\title{
FREE STATES OF THE GAUGE INVARIANT CANONICAL ANTICOMMUTATION RELATIONS. II
}

\author{
BY
}

B. M. BAKER

\begin{abstract}
A class of representations of the gauge invariant subalgebra of the canonical anticommutation relations (henceforth GICAR) is studied. These representations are induced by restricting the well-known pure, nongauge invariant generalized free states of the canonical anticommutation relations (henceforth CAR). Denoting a state of the CAR by $\omega$, and the unique generalized free state of the CAR such that $\omega\left(a(f)^{*} a(g)\right)=(f, T g)$ and $\omega(a(f) a(g))=(S f, g)$ by $\omega_{S, T}$, it is shown that a pure, nongauge invariant state $\omega_{S, T}$ induces a factor representation of the GICAR if and only if $\operatorname{Tr} T(I-T)=\infty$.
\end{abstract}

1. Introduction. The problem considered here is a natural extension of the work begun by the author in [2], where restrictions of gauge invariant generalized free states of the canonical anticommutation relations (CAR) to its $U(1)$ or gauge invariant subalgebra (GICAR) were studied. Viewing the CAR and GICAR as UHF and AF $C^{*}$-algebras in the sense of [5] and [4] respectively, a necessary and sufficient condition was obtained for such states to induce factor representations. The next simplest case is that of the nongauge invariant but pure generalized free state. Such states were analyzed by several authors [1], [3], the former directly generalizing a study of gauge invariant generalized free states [9]. In [6] the connection between this class of representations and the BCS theory of superconductivity is discussed.

Here, both the formalisms of [9] and [3] will be used when convenient, together with the obvious correspondence between them. The main result of this work, Theorem 4.17, is identical in conclusion to that of [2, Theorem 3.24]. In \$3, two complex structures are analyzed; analogous procedures are found in [1] and [11]. Throughout, the techniques and results of [8], [9], [4], and $[10]$ are relied upon heavily.

ACKNowledgement. I am indebted to Professor Robert T. Powers for his many valuable suggestions. I also thank John Phillips for some useful discussions.

Received by the editors December 12, 1977.

AMS (MOS) subject classifications (1970). Primary 46L05, 81A17; Secondary 81A81.

Key words and phrases. Anticommutation relations, gauge invariance, approximately finite $C^{*}$-algebra, generalized free states factor representations. 
2. Definitions, notation. Let $\mathcal{K}$ be a separable complex Hilbert space with orthonormal basis $\left\{f_{n}\right\}, n=1,2, \ldots$, and $\mathfrak{R}_{n}=\operatorname{span}\left\{f_{1}, \ldots, f_{n}\right\}$. The CAR algebra over $\mathfrak{T}_{n}$ is denoted by $\mathfrak{U}\left(\mathfrak{T}_{n}\right)$. It is constructed via a linear mapping $f \rightarrow a(f)$ satisfying the relations

$$
\begin{aligned}
a(f) a(g)+a(g) a(f) & =0, \\
a(f) a(g)^{*}+a(g)^{*} a(f) & =(g, f) I
\end{aligned}
$$

for all $f, g$ in $\Re_{n}$. We denote by $\mathfrak{A}(\mathcal{K})$ the CAR algebra over $\mathcal{K}$, the completion of $\cup_{n} \mathfrak{A}\left(\mathfrak{N}_{n}\right)$ written $\mathfrak{A}(\mathscr{K})=\overline{U_{n} \mathfrak{A}\left(\mathfrak{T}_{n}\right)}$. This is a UHF algebra in the sense of [5]. Note that commuting $2 \times 2$ matrix algebras are defined by the formulas $\mathscr{B}_{1}=\mathfrak{A}\left(\mathfrak{T}_{1}\right), \mathscr{B}_{k}=\mathfrak{A}\left(\mathfrak{T}_{k}\right) \cap \mathfrak{A}\left(\mathfrak{T}_{k-1}\right)^{c}, k=2,3, \ldots$; it follows that $\mathfrak{U}\left(\mathfrak{T}_{n}\right) \approx \otimes_{k=1}^{n} \mathscr{B}_{k}$, a $2^{n} \times 2^{n}$ matrix algebra (see e.g. [2]). We choose matrix units for the $\mathscr{\Re}_{k}$ as in[2]:

$$
\begin{array}{ll}
e_{11}^{(k)}=a\left(f_{k}\right) a\left(f_{k}\right)^{*}, & e_{21}^{(k)}=a\left(f_{k}\right) V_{k}, \\
e_{12}^{(k)}=a\left(f_{k}\right)^{*} V_{k}, & e_{22}^{(k)}=a\left(f_{k}\right)^{*} a\left(f_{k}\right)
\end{array}
$$

where

$$
\begin{aligned}
& V_{1}=I, \\
& V_{k}=\prod_{j=1}^{k-1}\left(I-2 a\left(f_{j}\right)^{*} a\left(f_{j}\right)\right), \quad k=2,3, \ldots
\end{aligned}
$$

We denote by $\mathfrak{A}^{\circ}(\mathscr{K})$ the $U(1)$ or gauge invariant $C^{*}$-subalgebra of $\mathfrak{A}(\mathcal{K})$. This is the algebra invariant under the automorphisms $\chi_{t}, 0<t<2 \pi$, defined by extending the map $a(f) \rightarrow e^{-i t} a(f)$ to all of $\mathfrak{U}(\mathscr{K})$. $\mathfrak{U}^{\circ}(\mathcal{K})$ is an AF algebra in the sense of [4].

Alternatively, the CAR algebra may be constructed over a real Hilbert space [3]. From $\mathscr{K}$ we obtain a real Hilbert space $(K, s)$ with scalar product $s($,$) : for each vector f \in \mathcal{K}$ there is a corresponding vector $[f] \in(K, s)$ and $s$ is given by

$$
s([f],[g])=\operatorname{Re}(f, g)
$$

which implies $s([f],[i f])=0$. As in the case of $\mathfrak{A}(\mathscr{K})$, an algebra $\mathfrak{A}(K, s)$ is constructed via a linear mapping $[f] \rightarrow u([f])$ satisfying the new relations

$$
\begin{gathered}
u([f]) u([g])+u([g]) u([f])=2 s([f],[g]) I, \\
u([f])^{*}=u([f])
\end{gathered}
$$

for all $[f],[g] \in(K, s)$. Now if $[f]$ and $[g]$ are real orthogonal, the algebra generated by $u([f]), u([g])$ over $\mathbf{C}$ is isomorphic to a (complex) $2 \times 2$ matrix algebra. Further, algebras $\mathfrak{Y}\left(M_{k}\right)$ and $B_{k}$ may be constructed analogously to $\mathfrak{U}\left(\mathfrak{M}_{k}\right)$ and $\mathfrak{B}_{k}$ above.

In the sequel we will investigate a class of representations of $\mathfrak{A}^{\circ}(\mathscr{K})$ obtained by restricting the pure nongauge invariant generalized free states of 
$\mathfrak{A}(\mathscr{K})$ to $\mathfrak{A}^{\circ}(\mathscr{K}) \subset \mathfrak{A}(\mathscr{K})$. A generalized free state $\omega$ of $\mathfrak{X}(\mathscr{K})(\mathfrak{X}(K, s))$ is completely determined by its values on monomials of the form $a(f)^{*} a(g)$, $a(f) a(g)(u([f]) u([g]))$ for all $f, g \in \mathscr{K}([f],[g] \in(K, s))$, see e.g. [7] and [3]. It follows that operators $S, T$ on $\mathscr{K}$ and $A$ on $(K, s)$ may be defined by the formulas:

$$
\omega\left(a(f)^{*} a(g)\right)=(f, T g), \quad \omega(a(f) a(g))=(S f, g)
$$

and

$$
\omega(u([f]) u([g]))=s([f],[g])+i s(A[f],[g]) .
$$

From the properties of a state, we find $T$ linear, $0<T<I, S$ antilinear, $S^{*}=-S$, and $A$ skew-adjoint, i.e. $A^{+}=-A$, with + denoting the real adjoint on $(K, s)$. We denote by $\omega_{S, T}\left(\omega_{A}\right)$ the generalized free state of $\mathfrak{A}(\mathcal{K})$ $(\mathfrak{A}(K, s))$ with the two-point functions given by $S, T(A)$ and the restriction of $\omega_{S, T}$ to $\mathfrak{A}^{\circ}(\mathcal{K})$ by $\omega_{S, T}^{\circ}$. Straightforward computation, using the isomorphism obtained by extending the mapping $u([f]) \rightarrow a(f)+a(f)^{*}$, yields, for a given state

$$
s(A[f],[g])=\operatorname{Re}((2 i S+i(2 T-I)) f, g) .
$$

It is a consequence of $[3$, Theorem 1] that a complex structure $J$ on $(K, s)$ induces a pure generalized free state $\omega_{J}$ of $\mathfrak{A}(K, s)$. Defining $\omega_{J_{1}}$ to be the state of $\mathfrak{X}(K, s)$ corresponding to the (pure) Fock state $\omega_{0,0}$ it follows directly from the above formula that the complex structure $J_{1}$ satisfies

$$
J_{1}[f]=-[i f], \quad J_{1}[i f]=[f] .
$$

A state of $\mathfrak{A}(\mathscr{K})$ is said to factorize with respect to the algebras $\mathscr{B}_{k}, k=0,1$, $2, \ldots$, above if $\omega(x y)=\omega(x) \omega(y)$ for $x \in \mathscr{B}_{k}, y \in \mathscr{B}_{l}, k \neq l$; we then write $\omega=\otimes{ }_{k=0}^{\infty} \omega \mid \mathscr{B}_{k}$. If the $\Re_{k}$ are (simultaneous) invariant subspaces for $S$ and $T$, it follows from the properties of a generalized free state that $\omega$ factorizes with respect to the associated $\mathscr{B}_{k}$; similarly if $\boldsymbol{M}_{k}$ are invariant subspaces for $\mathfrak{A}(K, s)$, then $\omega_{A}$ factorizes on the associated $B_{k}$. For further elaboration of notation, definitions, or results see [7], [2]-[4].

3. Factor condition. We begin with a proposition indicating the correspondence between pure generalized free states of $\mathfrak{A}(K, s)$ and complex structures on $(K, s)$; this is essentially [3, Theorem 3].

Proposition 3.1. Let $\omega_{A}$ be a generalized free state of $\mathfrak{X}(K, s)$. Then $\omega_{A}$ is pure $\Leftrightarrow A$ is a complex structure on $(K, s)$.

Proof. Since $\omega_{A}$ is a generalized free state $A^{+}=-A$; hence we need only show $A^{2}=-I$. In [3, Theorem 3] it is concluded that $\omega_{A}$ is pure $\Rightarrow A^{2}=$ $-I$ assuming $\operatorname{Ker}(A)$ has even or infinite dimension, and that $A^{2}=-I \Rightarrow \omega_{A}$ is pure. Thus we need only consider the case of odd, finite dimensional kernel. 
In this case we show $\omega_{A}$ is not pure. Choose a unit vector $[f] \in \operatorname{Ker}(A)$ and define $M=\operatorname{span}([f])$. Straightforward computation shows $\omega_{A}$ factorizes with respect to $\mathfrak{A}(M, s)$ and $\mathfrak{A}\left(M^{\perp}, s\right)$. Assuming $\omega_{A}$ pure, it follows from the proof of [7, Theorem 5.5] that $\omega_{A} \mid \mathfrak{A}(M, s)$ and $\omega_{A} \mid \mathfrak{A}\left(M^{\perp}, s\right)$ are also pure. Now $\mathfrak{A}(M, s)$ is a two-dimensional $C^{*}$-algebra generated by $I$ and $u([f])$ with two ideals generated by $I \pm u(f)$; it follows that the pure states satisfy $\omega(u([f]))= \pm 1$. However, for any generalized free state $\omega_{A}(u[f])=0$ contradicting the hypothesis that $\omega_{A}$ is pure with $\operatorname{Ker}(A)$ finite dimensional and odd. Done.

REMARK. The argument above shows $\omega_{A}$ pure $\Rightarrow \operatorname{ker}(A)=0$.

We will now consider an arbitrary pure generalized free state on $\mathfrak{A}(K, s)$ and its associated complex structure, call it $J_{2}$. The aim is to factorize $\omega_{J_{2}}$ by choosing finite dimensional subspaces of $(K, s)$ which are simultaneously invariant under $J_{2}$ and the complex structure $J_{1}$ of $\S 1$.

LEMMA 3.2. Let $J_{2}$ be an arbitrary complex structure on $(K, s)$ and $J_{1}$ be as above (see \$1). Then the operator $M=-\left(J_{1}-J_{2}\right)^{2}$ is a real selfadjoint operator on $(K, s)$ with the properties

(i) $0<M / 4<I$,

(ii) $\left[J_{1}, J_{2}\right]_{+}=M-2 I$.

Proof. Since $\left(J_{1}-J_{2}\right)^{+}=-\left(J_{1}-J_{2}\right), M$ is clearly selfadjoint. Further,

$$
0<\left(J_{1}-J_{2}\right)^{+}\left(J_{1}-J_{2}\right)=-\left(J_{1}-J_{2}\right)^{2}
$$

and

$$
\left\|-\left(J_{1}-J_{2}\right)^{2}\right\|=\left\|J_{1}-J_{2}\right\|^{2} \leqslant\left(\left\|J_{1}\right\|+\left\|J_{2}\right\|\right)^{2}=4
$$

giving (i). For (ii), simply multiply out $-\left(J_{1}-J_{2}\right)^{2}$. Done.

We now specialize to the case where $M$ has pure point spectrum.

Definition 3.3. Suppose $M / 4$ has pure point spectrum $\left\{\mu_{n}\right\}, n=1$, $2, \ldots$; note by $3.2,0 \leqslant \mu_{n} \leqslant 1$. We define $\theta_{n}$ by the formula

$$
\theta_{n}=\cos ^{-1}\left(1-2 \mu_{n}\right)
$$

The following proposition obtains simultaneous invariant subspaces for $J_{1}, J_{2}$ on which these operators take a simple form:

Proposition 3.4. Let $M=-\left(J_{1}-J_{2}\right)^{2}$ as above, and $K_{\mu_{n}}$ be the spectral subspace of $K$ associated with the spectrum point $4 \mu_{n}$ of the operator $M$. Then there is a basis for $K_{\mu_{n}}$ satisfying

$$
\begin{aligned}
& J_{1} \mid K_{\mu_{n}}=\left(\begin{array}{rr}
0 & -1 \\
1 & 0
\end{array}\right) \otimes I \mu_{n}, \quad \mu_{n}=0,1, \\
& J_{2} \mid K_{\mu_{n}}= \begin{cases}\left(\begin{array}{rr}
0 & -1 \\
1 & 0
\end{array}\right) \otimes I_{0}, & \mu_{n}=0 \\
\left(\begin{array}{rr}
0 & 1 \\
-1 & 0
\end{array}\right) \otimes I_{1}, & \mu_{n}=1,\end{cases}
\end{aligned}
$$


where $I_{\mu_{n}}, I_{0}, I_{1}$ are identities on real Hilbert spaces whose dimensions equal the multiplicities of eigenvalues $\mu_{n}, 0,1$ respectively and

$$
\begin{array}{ll}
J_{1} \mid K_{\mu_{n}}=\left(\begin{array}{rr}
0 & I \\
-I & 0
\end{array}\right) \otimes I_{\mu_{n},} & 0<\mu_{n}<1, \\
J_{2} \mid K_{\mu_{n}}=\left(\begin{array}{rr}
0 & R \\
-R^{T} & 0
\end{array}\right) \otimes I_{\mu_{n},} & 0<\mu_{n}<1,
\end{array}
$$

where $0, I, R$ represent $2 \times 2$ matrices, $0, I$ as usual and

$$
R=\left(\begin{array}{cc}
\cos \theta_{n} & -\sin \theta_{n} \\
\sin \theta_{n} & \cos \theta_{n}
\end{array}\right)
$$

with $\theta_{n}$ defined as in 3.3.

Proof. The basis for this proof is to make the ansatz implicit in (i) and (ii) and carry out the computations.

(i) Choose a unit vector $v$ in $K_{0}$; if there are none, let $I_{0}$ be the identity on a zero-dimensional space. Since $v$ is in $K_{0}$, we have $-\left(J_{1}-J_{2}\right)^{2} v=0$ and straightforward computation shows $-\left(J_{1}-J_{2}\right)^{2} J_{i} v=0$, hence $J_{i} v$ in $K_{0}$, $i=1$, 2. Clearly, $\left(v, J_{i} v\right)=0$ and $\left\|J_{i} v\right\|=\|v\|=1, i=1$, 2. Finally, using $\left[J_{1}, J_{2}\right]_{+}=-2 I$ we have $-\left(J_{1} J_{2} v, v\right)=\left(v, J_{1} J_{2} v\right)+2$ or $+1=\left(J_{2} v, J_{1} v\right)$ giving $J_{2} v=J_{1} v$. Hence, $v, J_{1} v$ are orthonormal and $J_{1}$ and $J_{2}$ are given by $2 \times 2$ matrices of the form appearing in (i). Now choose $w$ in the orthogonal complement of $v, J_{1} v$ and repeat the process for $w, J_{1} w$. Continuing until $K_{0}$ is exhausted, we obtain (i), for $\mu_{n}=0$. Similar computations to those above complete (i).

(ii) Given a unit vector $v_{1}$ in $K_{\mu_{n}}$, we wish to find four orthonormal vectors $v_{1}, v_{2}, v_{3}, v_{4}$ such that the following are satisfied:
(1) $J_{1} v_{1}=-v_{3}$,
(5) $J_{2} v_{1}=-v_{3} \cos \theta+v_{4} \sin \theta$,
(2) $J_{1} v_{2}=-v_{4}$,
(6) $J_{2} v_{2}=-v_{3} \sin \theta-v_{4} \cos \theta$,
(3) $J_{1} v_{3}=v_{1}$,
(7) $J_{2} v_{3}=v_{1} \cos \theta+v_{2} \sin \theta$,
(4) $J_{1} v_{4}=v_{2}$
(8) $J_{2} v_{4}=-v_{1} \sin \theta+v_{2} \cos \theta$,

suppressing the subscript $n$ on $\theta$ for convenience. Clearly, we can choose an arbitrary unit vector $v_{1}$ in $K_{\mu_{n}}$ (if not, let $I_{\mu_{n}}$ be the identity on a zero-dimensional space), and invert (3) to get $v_{3}=-J_{1} v_{1}$; substitute in (7) to get

$$
v_{2}=\left(I \cot \theta+J_{1} J_{2} \csc \theta\right) v_{1}
$$

and in (5) to obtain

$$
v_{4}=\left(-J_{1} \cot \theta+J_{2} \csc \theta\right) v_{1} \text {. }
$$

(Note $0<\mu_{n}<1$ implies $\sin \theta \neq 0$.) We now claim $v_{i}, i=1,2,3,4$, are orthonormal and satisfy (1) through (8) above. Given this, we may choose $w_{1}$ in the orthogonal complement of $v_{1}, i=1, \ldots, 4$, and repeat the process, 
obtaining (ii). Straightforward computation using $J_{i}^{+}=-J_{i}, J_{i}^{2}=-I, i=$ 1,2 , and $\left[J_{1}, J_{2}\right]_{+} \mid K_{\mu_{n}}=-2 \cos \theta_{n} I$ shows that the $v_{i}, i=1,2,3,4$, are in $K_{\mu_{n}}$, are orthonormal, and do satisfy (1) through (8). Done.

THEOREM 3.5. Let $(K, s)$ be the real Hilbert space corresponding to $\mathcal{K}$ as in $\S 1$, and $\mathfrak{A}(K, s)$ be the $C A R$ algebra over $(K, s)$. Let $\omega_{J_{1}}, \omega_{J_{2}}$ be the Fock state and an arbitrary pure generalized free state on $\mathfrak{A}(K, s)$ respectively. Suppose that $-\left(J_{1}-J_{2}\right)^{2}$ has pure point spectrum. Then we can write

$$
K=\bigoplus_{n=0}^{\infty} K_{\mu_{n}}
$$

with the $K_{\mu_{n}}$ satisfying the following:

(i) the $K_{\mu_{n}}$ are mutually orthogonal,

(ii) $\operatorname{dim}\left(K_{\mu_{n}}\right)=4,0<\mu_{n}<1$,

(iii) $\operatorname{dim}\left(K_{\mu_{n}}\right)=2, \mu_{n}=0,1$,

(iv) $\omega_{J_{2}}=\bigotimes_{r=0}^{\infty} \omega_{J_{2}} \mid B_{r}$,

where $B_{r}$ is a finite-dimensional matrix algebra, $r=1,2, \ldots$ In particular $\omega_{J_{2}}$ factorizes with respect to the $\left\{K_{\mu_{n}}\right\}, n=1,2, \ldots$

Proof. By hypothesis $-\left(J_{1}-J_{2}\right)^{2}$ has pure point spectrum which we denote $\left\{4 \mu_{n}\right\}, n=1,2, \ldots$, as above. Note by $3.2,0 \leqslant \mu_{n}<1$. Letting $K_{\mu_{n}}$ be the spectral subspace of $-\left(J_{1}-J_{2}\right)^{2}$ associated with the eigenvalue $4 \mu_{n}$, we choose a basis for these as in 3.4. This yields two and four dimensional mutually orthogonal invariant subspaces for $J_{i}, i=1,2$, corresponding to $\mu_{n}=0,1$ and $0<\mu_{n}<1$ respectively. Consequently, we may define $M_{r}=$ $\bigoplus_{n=0}^{r} K_{\mu_{n}}, B_{r}=\mathfrak{U}\left(M_{r+1}, s\right) \cap \mathfrak{U}\left(M_{r}, s\right)^{c}$ and obtain factorization for $\omega_{J_{2}}$ on the finite-dimensional $\left\{B_{r}\right\}, r=1,2, \ldots$ Done.

COROLlaRY 3.6. Let $\omega_{S, T}$ be a pure generalized free state of $\mathfrak{A}(\mathscr{H})$, and suppose $T$ has pure point spectrum. Then we can write

$$
\mathcal{K}=\bigoplus_{n=0}^{\infty} \mathcal{K}_{\mu_{n}}
$$

with the $\mathcal{K}_{\mu_{n}}$ satisfying the following:

(i) the $\mathscr{K}_{\mu_{n}}$ are mutually orthogonal,

(ii) $\operatorname{dim}\left(\mathscr{F}_{\mu_{n}}\right)=2,0<\mu_{n}<1$,

(iii) $\operatorname{dim}\left(\mathcal{K}_{\mu_{n}}\right)=1, \mu_{n}=0,1$,

(iv) $\omega_{S, T}=\otimes_{r=0}^{\infty} \omega_{S, T} \mid \mathscr{B}_{r}$ with $\mathscr{B}_{r}$ finite-dimensional matrix algebras; in particular $\omega_{s, T}$ factorizes with respect to the $\left\{\mathcal{K}_{\mu_{n}}\right\}, n=1,2, \ldots$,

(v) defining

$$
\Omega_{i j}^{(n)}= \begin{cases}\omega_{S, T}\left(e_{i_{n} j_{n}}^{(n)}\right), & \mu_{n}=0,1, \\ \omega_{S, T}\left(e_{i_{1} j_{1}}^{(n 1)} e_{i_{2} j_{2}}^{(n 2)}\right), & 0<\mu_{n}<1,\end{cases}
$$


with the arguments chosen to be the usual matrix units for

$$
\mathfrak{B}_{r}=\mathfrak{U}\left(\mathfrak{K}_{r+1}\right) \cap \mathfrak{U}\left(\mathfrak{N}_{r}\right)^{c}
$$

(and $\mathfrak{R}_{r}=\bigoplus_{n=0}^{r} \mathcal{K}_{\mu_{n}}$ ) we have

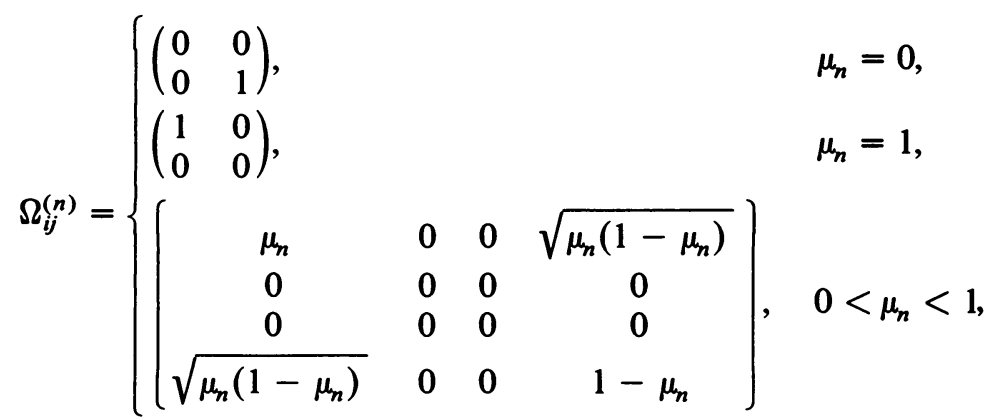

in matrix form.

PRoof. Consider $\omega_{J_{2}}$ on $\mathfrak{X}(K, s)$ corresponding to $\omega_{S, T}$ above. Straightforward computation (see $\S 1$ ) shows the operators $S, T$ on $\mathcal{K}$ correspond to $-\frac{1}{4}\left[J_{1}, J_{2}\right]_{-}, I / 2-\frac{1}{4}\left[J_{1}, J_{2}\right]_{+}$respectively. Hence $\left[J_{1}, J_{2}\right]_{+}$and $-\left(J_{1}-J_{2}\right)^{2}$ have pure point spectrum, since $T$ does. Invoking Proposition 3.4, we can get simultaneous invariant subspaces $K_{\mu_{n}}$ for $J_{i}, i=1,2$. Now with the $(K, s)$ to $\mathcal{K}$ correspondence, we obtain subspaces $\mathcal{K}_{\mu_{n}}$ for $K_{\mu_{n}}$; these are two or one (complex) dimensional given $K_{\mu_{n}}$ four or two (real) dimensional respectively, i.e. $0<\mu_{n}<1$ or $\mu_{n}=0,1$ respectively. By construction, the $\mathcal{K}_{\mu_{n}}$ are $S, T$ invariant, and $\omega_{S, T}$ therefore factorizes on the associated $\mathscr{B}_{n}$ yielding (i), (ii) and (iii). Finally, explicit computation gives (iv). Done.

LEMMA 3.7. Let $\omega=\omega_{S, T}$ be a pure generalized free state of $\mathscr{A}(\mathcal{K})$, such that $\operatorname{Tr} T(I-T)<\infty$. Let $(\Pi, \mathcal{H}, f)$ be the representation induced by $\omega$ via the GNS construction, and define

as in 3.6 and

$$
N_{n}=N\left(\mathscr{T}_{n}\right)=N\left(\bigoplus_{r=0}^{n} \mathscr{K}_{\mu_{r}}\right)
$$

$$
V_{n}=N_{n}-\omega\left(N_{n}\right) I .
$$

Then the sequence of operators $\left\{e^{i \Pi\left(V_{n}\right) t}\right\}, n=1,2, \ldots$, converges strongly to an isometry, call it $U_{t}$, on the closure of $\Pi\left(\mathfrak{A}^{\circ}(\mathcal{K})\right) f$, call it $\mathcal{H}^{\circ}$, for all $t$ in $(0,1 / 4)$.

PROof. We note that $V_{n}$ is selfadjoint, and $e^{i \Pi\left(V_{n}\right) t}$ is unitary for all integers $n$ and real numbers $t$. Hence if the limit exists on $\mathcal{H}^{\circ}$, it will be an isometry. Since convergence on a dense set in $\mathcal{H}^{\circ}$ allows us to extend to an isometry on all of $\mathcal{H}^{\circ}$, we need only show the former. Since $\mathcal{H}^{\circ}$ is complete, this will 
follow if the sequence

$$
\left\{e^{i \Pi\left(V_{n}\right) t} g\right\}, \quad n=1,2, \ldots,
$$

is Cauchy for all $g$ in a dense set. We first show that

(i) $\left\{e^{i \Pi\left(V_{n}\right) t} f\right\}, n=1,2, \ldots$,

is Cauchy, with $f$ the cyclic vector and finally that

(ii) $\left\{e^{i \Pi\left(V_{n}\right) t} \Pi(x) f\right\}, n=1,2, \ldots$,

is Cauchy for all $x$ in $\mathfrak{A}^{\circ}(\mathcal{K})$. Since $\Pi\left(\mathfrak{U}^{\circ}(\mathcal{K})\right)$ is dense in $\mathcal{K}^{\circ}$, the proof will be complete. To show (i) we first note that

$$
\left\|e^{i \Pi\left(V_{n}\right) t} f-e^{i \Pi\left(V_{n}\right) t} f\right\|^{2}=2-2 \operatorname{Re}\left(f, e^{i \Pi\left(V_{n}\right) t} f\right) .
$$

Using the factorization

$$
\omega_{S, T}=\bigotimes_{r=0}^{\infty} \omega_{S, T} \mid \Re_{r}
$$

of 3.6, we begin the computation. Recall

$$
\mathcal{K}=\bigoplus_{n=0}^{\infty} \mathcal{K}_{\mu_{n}}
$$

let $f_{k}$ be a basis for $\mathscr{K}_{\mu_{k}}, \mu_{k}=0,1$, and $f_{k}^{(1)}, f_{k}^{(2)}$ be a basis for $\mathscr{K}_{\mu_{k}}, 0<\mu_{k}<1$. Analogously we define

$$
n_{k}= \begin{cases}e_{22}^{(k)}, & \mu_{k}=0,1, \\ e_{22}^{(k 1)}+e_{22}^{(k 2)}, & 0<\mu_{k}<1,\end{cases}
$$

and observe that in matrix form

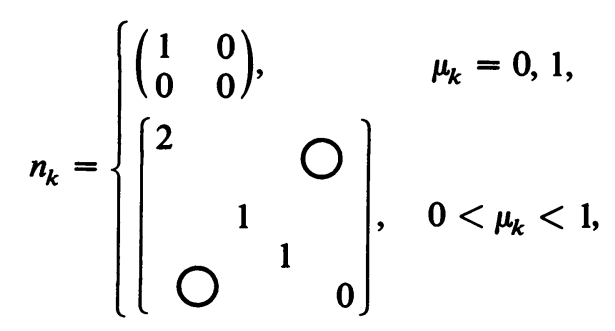

ignoring the rest of the tensor product, which are repeated identity matrices. Thus

$$
V_{n}-V_{m}=\sum_{k=m+1}^{n}\left(n_{k}-\omega\left(n_{k}\right) I\right)
$$

and

$$
e^{i\left(V_{n}-V_{m}\right) t}=\prod_{k=m+1}^{n} e^{i t\left(n_{k}-\omega\left(n_{k}\right) I\right)}
$$

for $\left[n_{k}, n_{j}\right]_{-}=0$. Since $\omega$ factorizes with respect to $\left\{\mathcal{K}_{\mu_{n}}\right\}, n=1,2, \ldots$, we 
also have

$$
\omega\left(e^{i\left(V_{n}-V_{m}\right) t}\right)=\prod_{k=m+1}^{n}\left(e^{i t\left(n_{k}-\omega\left(n_{k}\right) I\right)}\right) .
$$

Now using the matrix form of $\omega \mid \Re_{n}$ (namely $\Omega_{i j}^{(n)}$ of 3.6) and $n_{k}$, and straightforwardly computing we obtain

$$
\omega\left(e^{i\left(V_{n}-V_{m}\right) t}\right)=\prod_{k=m+1}^{n}\left\{\left(1-\mu_{k}\right) e^{-2 i t \mu_{k}}+\mu_{k} e^{2 i t\left(1-\mu_{k}\right)}\right\}
$$

Defining

$$
\omega\left(e^{i\left(V_{n}-V_{m}\right) t}\right)=\prod_{k=m+1}^{n}\left(1+z_{k}(t)\right)
$$

we have

$$
\begin{aligned}
\lim _{m, n \rightarrow \infty} \omega\left(e^{i\left(V_{n}-V_{m}\right) t}\right) & =1 \Leftrightarrow \prod_{k=0}^{\infty}\left(1+z_{k}(t)\right) \text { converges } \\
& \Leftrightarrow \sum_{k=0}^{\infty}\left|z_{k}(t)\right|<\infty
\end{aligned}
$$

by standard infinite product results. Direct computation shows that

$$
\left|z_{k}(t)\right|^{2}=-2\left(1-\mu_{k}\right) \cos \left(2 t \mu_{k}\right)-2 \mu_{k} \cos \left(2 t\left(1-\mu_{k}\right)\right) \text {. }
$$

We claim there exists an integer $N$ such that

$$
\left|z_{k}(t)\right|^{2} \leqslant 4 t \mu_{k}^{2}\left(1-\mu_{k}\right)^{2}
$$

for all $k>N$. To see this, first observe that if

$$
f^{\prime}(s) \leqslant g^{\prime}(s) \text { and } f(0)=g(0)=0
$$

(assuming $f$ and $g$ are differentiable with derivatives $f^{\prime}, g^{\prime}$ ) then

$$
\int_{0}^{t} f^{\prime}(s) d s<\int_{0}^{t} g^{\prime}(s) d s \text { or } f(t)<g(t) .
$$

Since $\left|z_{k}(0)\right|=0$, we need only show

$$
\frac{d}{d t}\left|z_{k}(t)\right|^{2}<4 \mu_{k}^{2}\left(1-\mu_{k}\right)
$$

for $k>N$, or

$$
-\sin (2 t)+\sin \left(2 t \mu_{k}\right)+\sin \left(2 t\left(1-\mu_{k}\right)\right)<\mu_{k}\left(1-\mu_{k}\right) .
$$

Now

$$
\operatorname{Tr} T(I-T)=2 \sum_{k=0}^{\infty} \mu_{k}\left(1-\mu_{k}\right)<\infty
$$

implies there exists an $N$ such that $\mu_{k}\left(1-\mu_{k}\right)<\frac{1}{4}$ for all $k>N$. Hence, there are two cases, $0<\mu_{k}<\frac{1}{2}$, and $\frac{1}{2}<\mu_{k}<1$, when $k>N$. The former gives 


$$
\begin{aligned}
-\sin (2 t)+\sin \left(2 t \mu_{k}\right)+\sin \left(2 t\left(1-\mu_{k}\right)\right) & <-\sin (2 t)+2 t \mu_{k}+\sin (2 t)=2 t \mu_{k} \\
& <\frac{1}{2} \mu_{k}<\mu_{k}\left(1-\mu_{k}\right)
\end{aligned}
$$

for all $t$ in $\left(0, \frac{1}{4}\right)$, since $x \leqslant \sin x$ and $\sin x$ is increasing for all $x$ in $(0, \pi / 2)$. Likewise, for the latter case we obtain

$$
-\sin (2 t)+\sin \left(2 t \mu_{k}\right)+\sin \left(2 t\left(1-\mu_{k}\right)\right)<\frac{1}{2}\left(1-\mu_{k}\right)<\mu_{k}\left(1-\mu_{k}\right)
$$

for all $t$ in $\left(0, \frac{1}{4}\right)$. Thus,

$$
\left|z_{k}(t)\right|<2 t^{1 / 2} \mu_{k}\left(1-\mu_{k}\right)<\mu_{k}\left(1-\mu_{k}\right)
$$

for all $k>N$ and $t$ in $\left(0, \frac{1}{4}\right)$. Therefore

$$
\begin{aligned}
\sum_{k=0}^{\infty} \mu_{k}\left(1-\mu_{k}\right) & <\infty \Rightarrow \sum_{k=0}^{\infty}\left|z_{k}(t)\right|<\infty \\
& \Rightarrow \prod_{k=0}^{\infty}\left(1+z_{k}(t)\right) \rightarrow a \neq 0 \Rightarrow \lim _{m, n \rightarrow \infty} \omega\left(e^{i\left(V_{n}-V_{m}\right) t}\right)=1 \\
& \Rightarrow \lim _{m, n \rightarrow \infty}\left\|e^{i \Pi\left(V_{m}\right) t} f-e^{i \Pi\left(V_{n}\right) t} f\right\|^{2}=0 \\
& \Rightarrow\left\{e^{i \Pi\left(V_{n}\right) t} f\right\}, \quad n=1,2, \ldots,
\end{aligned}
$$

is Cauchy for all $t$ in $\left(0, \frac{1}{4}\right)$. Now defining $\mathscr{D}=U_{k} \mathfrak{A}^{\circ}\left(\mathscr{T}_{k}\right)$ we have $\mathscr{D}$ dense in $\mathfrak{A}^{\circ}(\mathscr{K})$ and $\Pi(\mathscr{D}) f$ dense in $\Pi\left(\mathfrak{A}^{\circ}(\mathcal{K})\right) f$ dense in $\mathscr{H}^{\circ}$. Consequently, for (ii) we need only show

$$
\left\{e^{i \Pi\left(V_{n}\right) t} \Pi(x) f\right\}, \quad n=1,2, \ldots,
$$

is Cauchy for all $t$ in $\left(0, \frac{1}{4}\right)$ and $x$ in $\mathscr{D}$ to complete the proof. But $x$ in $\mathscr{D}$ implies $x$ in $\mathfrak{A}^{\circ}\left(\mathfrak{K}_{p}\right)$ for some $p$, and clearly $V_{n}$ is in $\mathscr{Z}\left(\mathfrak{U}^{\circ}\left(\mathfrak{T}_{n}\right)\right)$. Hence,

$$
\left[\Pi\left(V_{n}\right), \Pi(x)\right]_{-}=\left[e^{i \Pi\left(V_{n}\right) t}, \Pi(x)\right]_{-}=0
$$

for all $n \geqslant p$, and for all $m, n \geqslant p$ we have

$$
\left\|e^{i \Pi\left(V_{n}\right) t} \Pi(x) f-e^{i \Pi\left(V_{m}\right) t} \Pi(x) f\right\| \leqslant\|\Pi(x)\|\left\|e^{i \Pi\left(V_{n}\right) t} f-e^{i \Pi\left(V_{m}\right) t} f\right\| \rightarrow 0
$$

as $n, m \rightarrow \infty$. This completes (ii), and the lemma. Done.

REMARK. As $\left|z_{k}(t)\right|=\left|z_{k}(-t)\right|$, the proof of 3.7 gives strong convergence of $e^{i \Pi\left(V_{n}\right) t}$ for all $t$ in $\left(-\frac{1}{4}, \frac{1}{4}\right)$; thus (1) for fixed $t$ in this interval, the adjoint sequence also converges strongly and hence (2) the limiting $U_{t}$ are actually unitary.

LEMMA 3.8. Let $\omega=\omega_{S, T}$ be a pure generalized free state of $\mathfrak{X}(\mathscr{K})=\overline{\bigcup_{n} \mathfrak{X}\left(\mathfrak{T}_{n}\right)}$ and suppose $T$ has pure point spectrum. Given $A$ in $\mathfrak{X}\left(\mathfrak{T U}_{n}\right)$ define

$$
q_{A}(t)=\omega\left(A e^{i N_{n} t}\right)-\omega(A) \omega\left(e^{i N_{n} t}\right) .
$$

Then the Fourier components of $q_{A}$ are all even or all odd. 
Proof. We decompose $\mathscr{K}$ as in Corollary 3.6, and let $\mathscr{T}_{n}=\bigoplus_{r=0}^{n} \mathcal{K}_{\mu_{r}}$. Now we may compute $q_{A}(t)$ as a product of finite-dimensional traces. For the two-dimensional $\mathcal{K}_{\mu}$ we observe only even powers of $e^{i t}$ appear in the associated trace; for the one-dimensional $\mathcal{K}_{\mu}$, only odd powers appear. Hence we have two cases (1) $\mathfrak{K}_{n}$ contains an odd number of one-dimensional $\mathcal{K}_{\mu}$, (2) $\mathfrak{K}_{n}$ contains an even number of one-dimensional $\mathcal{K}_{\mu_{r}}$. In case (1) all Fourier components of $q_{A}(t)$ are seen to be odd, in case (2) all even. Done.

COROllaRY 3.9. Let $q_{A}(t)$ be as above. Then either

(i) $q_{A}(t)=\sum_{k=0}^{p} a_{k}\left(1-e^{2 i k t}\right)$ or

(ii) $q_{A}(t)=\sum_{k=0}^{q} b_{k}\left(1-e^{2 i k t}\right) e^{i t}$

with $a_{k}, b_{k}$ complex numbers and $p, q \leqslant n$.

Proof. By the above lemma, all Fourier components are odd or even, simultaneously. But by definition $q_{A}(0)=0$; this enables us to write

$$
q_{A}(t)=\sum_{k=0}^{r} c_{k}\left(1-e^{i k t}\right)
$$

with $r \leqslant 2 n$, since $\Re_{n}$ has at most $n$ two-dimensional subspaces. Combining these two facts we can define $a_{k}$ or $b_{k}$ to obtain (i) or (ii). Done.

We are now ready to prove

THEOREM 3.10. Let $\omega_{S, T}$ be a pure generalized free state of $\mathfrak{X}(\mathcal{K})$, and suppose $T$ has pure point spectrum. Suppose further that $T$ is not a projection. Then $\omega_{S, T}^{\circ}$ is a factor state $\Leftrightarrow \operatorname{Tr} T(I-T)=\infty$.

Proof. $(\Rightarrow)$ To show this, we suppose $\operatorname{Tr} T(I-T)<\infty$ and demonstrate that $\omega_{S, T}$ is not a factor. To this end we consider the $U_{t}$ of Lemma 3.7. Since $U_{t}$ is a strong limit of operators in $\Pi\left(\mathfrak{U}^{\circ}(\mathcal{K})\right)$, we have $U_{t}$ in $\Pi\left(\mathscr{U}^{\circ}(\mathcal{K})\right)^{\prime \prime}$ for all $t$ in $\left(0, \frac{1}{4}\right)$, which is the strong and weak closure of $\Pi\left(\mathfrak{U}^{\circ}(\mathcal{K})\right)$. Further, for all $x$ in $\mathscr{D}=\cup_{k} \mathfrak{A}^{\circ}\left(\mathscr{T R}_{k}\right)$ we saw that

$$
\left[e^{i \Pi\left(V_{n}\right) t}, \Pi(x)\right]_{-}=0
$$

for all $n \geqslant p$, with $x$ in $\mathfrak{U}^{\circ}\left(\mathscr{T}_{p}\right)$. Thus

$$
\lim _{n \rightarrow \infty}\left[e^{i \Pi\left(V_{n}\right) t}, \Pi(x)\right]_{-} g=0
$$

for all $g$ in $\mathcal{H}^{\circ}$. But then

$$
0=\lim _{n \rightarrow \infty}\left[e^{i \Pi\left(V_{n}\right) t}, \Pi(x)\right]_{-} g=\left[\lim _{n \rightarrow \infty} e^{i \Pi\left(V_{n}\right) t}, \Pi(x)\right]_{-} g
$$

for all $g$ in $\mathcal{H}^{\circ}$, since operator multiplication is continuous. Hence

$$
\left[U_{t}, \Pi(x)\right]_{-} g=0
$$


for all $g$ in $\mathcal{H}^{\circ}$ or

$$
\left[U_{t}, \Pi(x)\right]_{-}=0 .
$$

But $\Pi(\mathscr{D})$ is dense in $\Pi\left(\mathfrak{A}^{\circ}(\mathscr{K})\right)$ so

$$
\left[U_{t}, \Pi(y)\right]_{-}=0
$$

for all $y$ in $\mathfrak{A}^{\circ}(\mathscr{K})$, i.e., $U_{t}$ is in $\Pi\left(\mathfrak{H}^{\circ}(\mathscr{K})\right)^{\prime}$. Summarizing, we have $U_{t}$ in $\Pi\left(\mathfrak{A}^{\circ}(\mathcal{K})\right)^{\prime} \cap \Pi\left(\mathfrak{Y}^{\circ}(\mathscr{K})\right)^{\prime \prime}$ or $U_{t}$ in $\mathscr{Z}\left(\Pi\left(\mathfrak{A}^{\circ}(\mathscr{K})\right)\right)$ for all $t$ in $\left(0, \frac{1}{4}\right)$. To prove $\omega_{S, T}$ is not a factor we need only show $U_{t}$ is not a multiple of the identity. Since $U_{t}$ is unitary, this reduces to $U_{t} \neq e^{i \phi} I$; for this it is sufficient to show $\left(f, U_{\downarrow} f\right) \neq e^{i \phi}$. However,

$$
\begin{aligned}
\left(f, U_{t} f\right) & =\left(f, \lim _{n \rightarrow \infty} e^{i \Pi\left(V_{n}\right) t} f\right) \\
& =\lim _{n \rightarrow \infty}\left(f, e^{i \Pi\left(V_{n}\right) t} f\right)=\lim _{n \rightarrow \infty} \omega_{S, T}\left(e^{i V_{n} t}\right) \\
& =\prod_{k=1}^{\infty}\left\{\left(1-\mu_{k}\right)+\mu_{k} e^{2 i t}\right\} e^{-2 i t \mu_{k}}
\end{aligned}
$$

and therefore

$$
\lim _{n \rightarrow \infty}\left|\omega_{S, T}\left(e^{i V_{n} t}\right)\right|^{2}=\prod_{k=1}^{\infty}\left\{1-2 \mu_{k}\left(1-\mu_{k}\right)(1-\cos (2 t))\right\} .
$$

For all $t$ in $\left(0, \frac{1}{4}\right)$ we have

$$
\lim _{n \rightarrow \infty}\left|\omega_{S, T}\left(e^{i V_{n^{t}}}\right)\right|^{2}=a \neq 1
$$

by the standard infinite product result, given $\sum_{k=1}^{\infty} \mu_{k}\left(1-\mu_{k}\right) \neq 0$. If not, we have $\mu_{k}\left(1-\mu_{k}\right)=0$ for all $k$, and this implies $T$ is a projection, contradiction the hypothesis. Done $(\Rightarrow)$.

$(\Leftarrow)$ Following [2, Proposition 3.14], we define

$$
\begin{aligned}
q_{A}(t) & =\omega\left(A e^{i N_{n} t}\right)-\omega(A) \omega\left(e^{i N_{n} t}\right), \\
P_{m n}(t) & =\omega\left(e^{i\left(N_{m}-N_{n}\right) t}\right)
\end{aligned}
$$

with $A$ in $\mathfrak{A}^{\circ}\left(\mathscr{T}_{n}\right), m \geqslant n$, and the subscripts $S, T$ suppressed for convenience. Using the identical calculation, we conclude that

$$
\lim _{m \rightarrow \infty}\left\|q_{A}(t) P_{m n}(t)\right\|_{1}=0
$$

implies $\omega^{\circ}$ is a factor. As in [2], \|\|$_{1}$ denotes the Fourier-one norm. Since $n$ is fixed and finite, this is equivalent to

$$
\lim _{m \rightarrow \infty}\left\|q_{A}(t) P_{m}(t)\right\|_{1}=0
$$

defining $P_{m}(t)$ to be the product from 1 to $m$ and relabeling the $n$th term as the first. By Corollary 3.9 we can write 


$$
q_{A}(t) P_{m}(t)=\left\{\begin{array}{l}
\sum_{k=0}^{p} a_{k}\left(1-e^{2 i k t}\right) P_{m}(t), \\
\sum_{k=0}^{q} b_{k}\left(1-e^{2 i k t}\right) e^{i t} P_{m}(t)
\end{array}\right.
$$

and since for any function $f(t)$

$$
\left\|e^{i t} f(t)\right\|_{1}=\|f(t)\|_{1}
$$

it is sufficient to show

$$
\lim _{m \rightarrow \infty}\left\|\left(1-e^{2 i k t}\right) P_{m}(t)\right\|_{1}=0
$$

the total number of terms in either sum being finite. But now note that

$$
\left(1-e^{2 i k t}\right)=\sum_{s=0}^{k-1} e^{2 i s t}\left(1-e^{2 i t}\right)
$$

hence we need only show

$$
\lim _{m \rightarrow \infty}\left\|\left(1-e^{2 i t}\right) P_{m}(t)\right\|_{1}=0
$$

again due to the finiteness of the sum limit. Finally recall

$$
P_{m}(t)=\omega^{\circ}\left(\prod_{k=1}^{m} e^{i n_{k} t}\right)=\prod_{k=1}^{m} \omega^{\circ}\left(e^{i m_{k} t}\right)
$$

by the factorization properties of $\omega^{\circ}$. Now straightforward computation shows

$$
\omega^{\circ}\left(e^{i n_{k} t}\right)= \begin{cases}1, & \mu_{k}=0, \\ e^{i t}, & \mu_{k}=1, \\ \left(1-\mu_{k}\right)+\mu_{k} e^{2 i t}, & 0<\mu_{k}<1,\end{cases}
$$

and we observe the presence of the $\mu_{k}=0,1$ terms do not alter the Fourier-one norm, since they are modulus one. Thus

$$
\begin{aligned}
\lim _{m \rightarrow \infty} & \left\|\left(1-e^{2 i t}\right) P_{m}(t)\right\|_{1} \\
& =\lim _{m \rightarrow \infty}\left\|\left(1-e^{2 i t}\right) \prod_{\left\{k: 0<\mu_{k}<1\right\}}^{m}\left\{\left(1-\mu_{k}\right)+\mu_{k} e^{2 i t}\right\}\right\|_{1}=0
\end{aligned}
$$

since

$$
\sum_{k=1}^{\infty} \mu_{k}\left(1-\mu_{k}\right)=\infty \Rightarrow \sum_{\left\{k: 0<\mu_{k}<1\right\}}^{\infty} \mu_{k}\left(1-\mu_{k}\right)=\infty
$$

and the above Fourier-one norm goes to zero by the arguments of [2, Theorem 3.20] with $2 t$ replacing $t$. Done. 
4. This section is aimed at removing the point spectrum condition from Theorem 3.10. We begin by obtaining an estimate for $\left\|\omega_{J}-\omega_{J}\right\|$ in terms of $\left\|J-J^{\prime}\right\|_{\text {H.S. }}$.

Lemma 4.1. Let $\omega$ be a state of a $C^{*}$-algebra $\mathfrak{A}$, and $E_{1}, E_{2} \in \mathfrak{U}$ be commuting projections. Then if $\omega\left(E_{1}\right) \geqslant 1-\lambda$ and $\omega\left(E_{2}\right) \geq 1-\mu$ we have $\omega\left(E_{1} E_{2}\right)>1-\lambda-\mu$.

Proof. Note that

$$
E_{1} E_{2}+E_{1}\left(I-E_{2}\right)+E_{2}\left(I-E_{1}\right)+\left(I-E_{1}\right)\left(I-E_{2}\right)=I .
$$

Applying $\omega$ to each of the summands on the left we get four numbers, call them $a, b, c, d$ respectively; clearly $a, b, c, d>0$. Also

(i) $\omega(I)=1=a+b+c+d$,

(ii) $\omega\left(E_{1}\right)=a+b \geqslant 1-\lambda$,

(iii) $\omega\left(E_{2}\right)=a+c \geqslant 1-\mu$,

(iv) $\omega\left(E_{1} E_{2}\right)=a$.

Adding (ii) and (iii) gives $2 a+b+c \geqslant 2-\lambda-\mu$; thus $2+a+b+c+d$ $=1+a>2-\lambda-\mu$ or $\omega\left(E_{1} E_{2}\right)>1-\lambda-\mu$. Done.

Lemma 4.2 [9, Lemma 2.5]. Suppose $\omega_{1}$ and $\omega_{2}$ are states of a $C^{*}$-algebra. Suppose $\left\{E_{\gamma} ; \gamma \in I_{0}\right\}$ is a decreasing net of projections in $\mathfrak{A}$ (i.e. $E_{\alpha}<E_{\beta}$ for $\alpha>\beta)$ with the property that $\omega_{1}\left(E_{\gamma}\right)=1$ for all $\gamma \in I_{0}$, and if $\omega$ is any state of $\mathfrak{A}$ such that $\omega\left(E_{\gamma}\right)=1$ for all $\gamma \in I_{0}$, then $\omega=\omega_{1}$. Let $\alpha=\inf \left(\omega_{2}\left(E_{\gamma}\right) ; \gamma \in I_{0}\right)$. Then the following inequalities are valid:

$$
2(1-\alpha) \leqslant\left\|\omega_{1}-\omega_{2}\right\| \leqslant 2(1-\alpha)^{1 / 2} .
$$

Furthermore if $\omega_{2}$ is pure, then

$$
\left\|\omega_{1}-\omega_{2}\right\|=2(1-\alpha)^{1 / 2} .
$$

LEMMA 4.3. Let $\omega_{0,0}$ be the Fock state, and $\omega_{S, T}$ be a pure generalized free state on $\mathfrak{A}(\mathcal{K})$, the CAR algebra over a complex separable Hilbert space $\mathscr{K}$. Then

$$
\left\|\omega_{0,0}-\omega_{S, T}\right\|<2\|T\|_{T r}^{1 / 2}
$$

Proof. Let $\left\{f_{k}\right\}$ be an orthonormal basis for $\mathscr{K}$ and

$$
E_{N}=\prod_{k=1}^{N} a\left(f_{k}\right) a\left(f_{k}\right)^{*}=\prod_{k=1}^{N} e_{11}^{(k)}
$$

It follows from Lemma 4.2 and the properties of the Fock state that

$$
\left\|\omega_{0,0}-\omega_{S, T}\right\|=2(1-\alpha)^{1 / 2}
$$

with $\alpha=\lim _{N \rightarrow \infty} \omega_{S, T}\left(E_{N}\right)$. But $\omega_{S, T}\left(a\left(f_{k}\right) a\left(f_{k}\right)^{*}\right)=1-\left(f_{k}, T f_{k}\right)$, and we will 
show

$$
\omega_{S, T}\left(E_{N}\right) \geqslant 1-\sum_{k=1}^{N}\left(f_{k}, T f_{k}\right)
$$

by induction. Equality holds for $N=1$, so assume true for $N$; for $N+1$ we obtain

$$
\omega_{S, T}\left(E_{N+1}\right)=\omega_{S, T}\left(E_{N} e_{11}^{(N+1)}\right) \geqslant 1-\sum_{k=1}^{N}\left(f_{k}, T f_{k}\right)-\left(f_{N+1}, T f_{N+1}\right)
$$

by Lemma 4.1. Hence $\alpha \leqslant 1-\|T\|_{\mathrm{Tr}}$ and by Lemma 4.2

$$
\left\|\omega_{0,0}-\omega_{S, T}\right\|=2(1-\alpha)^{1 / 2}<2\|T\|_{\mathrm{Tr}}^{1 / 2} .
$$

Done.

LEMMA 4.4. Let $\omega_{S, T}, \omega_{S^{\prime}, T^{\prime}}$, be pure generalized free states of $\mathfrak{X}(\mathcal{K})$ and $\omega_{J}$, $\omega_{J}$, the corresponding states of $\mathfrak{U}(K, s)$ respectively. Then

$$
\frac{1}{8}\left\|J-J^{\prime}\right\|_{\text {H.S. }}^{2}=\left\|S-S^{\prime}\right\|_{\text {H.S. }}^{2}+\left\|T-T^{\prime}\right\|_{\text {H.S. }}^{2}
$$

with H.S. denoting the real Hilbert-Schmidt norm on $(K, s)$ on the left and H.S. denoting the standard Hilbert-Schmidt norm on $\mathcal{K}$ on the right.

Proof. Using the relation

$$
s(A[f],[g])=\operatorname{Re}((2 i S+i(2 T-I)) f, g)
$$

(see §1) we directly compute $\left\|J-J^{\prime}\right\|_{\text {H.S. }}^{2}$ with the basis $\left\{\left[f_{n}\right],\left[i f_{n}\right]\right\}$ of $\mathfrak{X}(K, s)$ to obtain the equality. Done.

Proposition 4.5. Let $J, J^{\prime}$ be complex structures on $(K, s)$ and $\omega_{J}, \omega_{J^{\prime}}$ the corresponding generalized free states of $\mathfrak{A}(K, s)$. Then

$$
\left\|J-J^{\prime}\right\|_{\text {H.S. }}<\sqrt{2} \varepsilon \Rightarrow\left\|\omega_{J}-\omega_{J}\right\|<\varepsilon .
$$

PROOF. Let $\omega_{L}$ be the pure generalized free state of $\mathfrak{U}(K, s)$ corresponding to the state $\omega_{0,0}$ of $\mathfrak{A}(\mathscr{K})$. Straightforward computation shows that $L$ is a complex structure on $(K, s)$. Since any two complex structures may be related by an orthogonal transformation $B$, and the mapping $\tau_{B}(u(h))=u(B h)$ preserves the anticommutation relations and thus extends to an automorphism of $\mathfrak{U}(K, s)$, there is an automorphism $\tau_{B}$ of $\mathfrak{U}(K, s)$ such that $\omega_{J} \circ \tau_{B}=$ $\omega_{L}$. Hence $\omega_{J^{\prime}} \circ \tau_{B}=\omega_{M}$ with $M=B^{+} J^{\prime} B$, a complex structure. Let $\omega_{S, T}$ on $\mathfrak{U}(\mathscr{K})$ be the state corresponding to $\omega_{M}$ on $\mathfrak{U}(K, s)$. It follows from Lemma 4.3 that

$$
\left\|\omega_{J}-\omega_{J}\right\|=\left\|\omega_{L}-\omega_{M}\right\|=\left\|\omega_{0,0}-\omega_{S, T}\right\| \leqslant 2\|T\|_{T_{r}^{1 / 2}}^{1 / 2}
$$

Since $M^{2}=-I$ on $(K, s)$ we have the corresponding equation on $\mathscr{K}$ :

$$
(2 i S+i(2 T-I))^{2}=-I
$$


or

$$
-S^{2}-S T+T S+T^{2}-T=0 .
$$

Let $\left\|J-J^{\prime}\right\|_{\text {H.S. }}^{2}<\sqrt{2} \varepsilon$; by Lemma 4.4

$$
2 \varepsilon^{2}>\left\|J-J^{\prime}\right\|_{\text {H.S. }}^{2}=\|L-M\|_{\text {H.S. }}^{2}=8\left(\|S\|_{\text {H.S. }}^{2}+\|T\|_{\text {H.S. }}^{2}\right) \text {. }
$$

We conclude $S$ and $T$ are Hilbert-Schmidt class, hence $S T$ and $T S$ are trace class. From the above identity, we observe that $T$ is trace class. Taking the trace of both sides and noting $T>0$, we obtain

$$
\|S\|_{\text {H.S. }}^{2}+\|T\|_{\text {H.S. }}^{2}=\|T\|_{T r}<\varepsilon^{2} / 4
$$

and $\left\|\omega_{J}-\omega_{J}\right\|<\varepsilon$. Done.

Definition 4.6. Let $\left\{\left[f_{n}\right],\left[i f_{n}\right]\right\}$ be a basis for $(K, s)$ and $J_{1}$ the complex structure associated with this basis as in $\S 1$. Let $J_{2}$ be an arbitrary complex structure on $(K, s)$ and $M=-\left(J_{1}-J_{2}\right)^{2}$ as in Lemma 3.2. We define subspaces $K_{0}, K_{1}, K_{2}$ of $(K, s)$ and an operator $\Lambda$ on $(K, s)$ by the formulas

$$
\begin{aligned}
& K_{0}=\{h \in K: M h / 4=0\}, \quad \Lambda \mid K_{0}=0, \\
& K_{1}=\{h \in K: M h / 4=h\}, \quad \Lambda \mid K_{1}=\pi I, \\
& K_{2}=K \ominus\left(K_{0} \oplus K_{1}\right), \quad \Lambda \mid K_{2}=\cos ^{-1}\left(I+\frac{1}{2}\left(J_{1}-J_{2}\right)^{2}\right) .
\end{aligned}
$$

REMARK. It follows directly from the definitions that

$$
M=2 I-2 \cos \Lambda \text {. }
$$

The following definitions aim toward constructing a continuous realization of $J_{1}, J_{2}$ and $\Lambda_{2}$ as multiplication by matrices of functions on a measure space. An application of the Weyl-von Neumann theorem will then yield an appropriate sequence of complex structures approaching $J_{2}$ in HilbertSchmidt norm.

Definition 4.7. Let $\Lambda_{2}=\int_{0}^{\pi} \lambda d E_{\lambda}$, the spectral resolution of $\Lambda_{2}$. Let $f_{0}$ be a unit vector in $K_{2}$. We define a measure $\mu_{0}$ on $[0, \pi]$ by the formula

$$
\mu([0, \lambda])=\left(f_{0}, E_{\lambda} f_{0}\right)
$$

Definition 4.8. Let $h$ be in $\mathcal{K}_{0}=\mathfrak{C}_{\mathbf{R}}^{2}\left([0, \pi], \mu_{0}\right) \otimes \mathbf{R}^{4}$, i.e. $h$ is a 4-tuple with components $h_{i}$ real, $\mu_{0}$ square-integrable functions on $[0, \pi], i=1, \ldots, 4$. We define a mapping

$$
U_{0}: \mathcal{K}_{0} \rightarrow K_{2}
$$

by the formula

$$
\begin{aligned}
U_{0} h= & \int_{0}^{\pi} h_{1}(\lambda) d E_{\lambda} f_{0}+\int_{0}^{\pi} h_{2}(\lambda)\left[\csc \lambda\left(I \cos \lambda+J_{1} J_{2}\right)\right] d E_{\lambda} f_{0} \\
& +\int_{0}^{\pi} h_{3}(\lambda)\left(-J_{1}\right) d E_{\lambda} f_{0}+\int_{0}^{\pi} h_{4}(\lambda)\left[\csc \lambda\left(-J_{1} \cos \lambda+J_{2}\right)\right] d E_{\lambda} f_{0}
\end{aligned}
$$


REMARK. This mapping is clearly linear. It is also well defined when the component functions $h_{i}$ vanish in a neighborhood of 0 and $\pi$. Further, with the inner product $\langle h, g\rangle_{0}=\Sigma_{i=1}^{4} \int_{0}^{\pi} h_{i}(\lambda) g_{i}(\lambda) d \mu_{0}(\lambda)$ it is straightforward but tedious to show that $U_{0}$ is isometric, using the properties of the spectral integral and calculations analogous to those showing $v_{1}, v_{2}, v_{3}, v_{4}$ orthonormal outlined in the proof of Proposition 3.4. Since $U_{0}$ is an isometry it may be extended to arbitrary square integrable $h_{i}$ by continuity; hence $U_{0}$ is well defined.

Definition 4.9. Let $f_{0}$ be a unit vector in $K_{2}$, and $P\left[J_{1}, J_{2}\right]$ be the polynomial algebra generated by $J_{1}, J_{2}$. We define $H_{0}=\left[P\left[J_{1}, J_{2}\right] f_{0}\right]^{-}$.

LEMMA 4.10. $H_{0} \subset K_{2}$.

Proof. By straightforward computation (see the proof of Proposition 3.4) we observe $J_{i} K_{0} \subset K_{0}$ and $J_{i} K_{1} \subset K_{1}, i=1,2$. Hence for all $g \in K_{0}\left(K_{1}\right)$ we have $s\left(g, J_{i} f_{0}\right)=s\left(-J_{i} g, f_{0}\right)=0$. Thus $J_{i} f_{0} \in K_{2}$ and it follows that $\left[P\left[J_{1}, J_{2}\right] f_{0}\right]^{-} \subset K_{2}$. Done.

REMARK. By the formula defining $U_{0}$ and Lemma 4.10 we conclude $U_{0} h \in H_{0}$. We may now construct a sequence of measures on $[0, \pi],\left\{\mu_{k}\right\}$, $k=0,1,2, \ldots$, and an isometry $U: \bigoplus_{k} \mathcal{H}_{k} \rightarrow K_{2}$ with $\mathcal{H}_{k}=\mathfrak{L}_{\mathrm{R}}^{2}([0, \pi]$, $\left.\mu_{k}\right) \otimes \mathbf{R}^{4}$. Let $f_{0}$ be a unit vector in $K_{2}$ as above; we define $U_{0}: \mathcal{H}_{0} \rightarrow H_{0} \subset$ $K_{2}$ as above. Now choose a unit vector $f_{1} \in H_{0}^{\perp} \cap K_{2}$. Define $\mu_{1}([0, \lambda])=$ $\left(f_{1}, E_{\lambda} f_{1}\right), H_{1}=\left[P\left[J_{1}, J_{2}\right] f_{1}\right]^{-}$, and $U_{1}$ analogously to $U_{0}$; by the same argument $U_{1}$ is also an isometry. Repeating this process we must exhaust $K_{2}$ in at most countably many steps since we assume $K_{2}$ separable. That is, $K_{2}=\bigoplus_{k} H_{k}$ with $k$ running over a finite or countable set. We now give

Definition 4.11. Let $U_{k}$ and $\mathcal{H}_{k}$ be as above, and $\mathcal{H}=\bigoplus_{k} \mathcal{H}_{k}$. We define an isometry $U: \mathcal{H} \rightarrow K_{2}$ by the formulas $U \mid \mathcal{H}_{k}=U_{k}$.

We are now ready to realize $J_{1}$ and $J_{2}$ on $K_{2}$ via operators $J_{1}^{\prime}$ and $J_{2}^{\prime}$ on $\mathcal{H}$.

DeFinition 4.12. We define the operators $J_{1}^{\prime}$ and $J_{2}^{\prime}$ on $\mathcal{H}$ by the formulas

$$
J_{1}^{\prime}\left|\mathscr{H}_{k}=\left(\begin{array}{cc}
0 & I \\
-I & 0
\end{array}\right), \quad J_{2}^{\prime}\right| \mathcal{H}_{k}=\left(\begin{array}{cc}
0 & R \\
-R^{T} & 0
\end{array}\right)
$$

where

$$
I=\left(\begin{array}{ll}
1 & 0 \\
0 & 1
\end{array}\right)
$$

0 and 1 denoting multiplication by the zero and unit functions on $[0, \pi]$ respectively and

$$
R=\left(\begin{array}{cr}
\cos \lambda & -\sin \lambda \\
\sin \lambda & \cos \lambda
\end{array}\right)
$$

each entry denoting multiplication by the given function.

LeMma 4.13. Let $U, J_{i}, J_{i}^{\prime}, i=1,2$, be as above. Then $U J_{i}^{\prime}=J_{i} U, i=1,2$. 
Proof. Choose $h \in \mathcal{H}$, then $h=\Sigma_{k} h_{k}, h_{k} \in \mathcal{H}_{k}$. It is clearly sufficient to show the formula holds for $h \in \mathcal{F}_{k}=\mathscr{L}_{\mathbf{R}}^{2}\left([0, \pi], \mu_{k}\right) \otimes \mathbf{R}^{4}$, so let

$$
h=\left(\begin{array}{c}
h_{1}(\lambda) \\
0 \\
0 \\
0
\end{array}\right) \in \mathcal{K}_{k}
$$

Then

$$
\begin{aligned}
U J_{2}^{\prime}\left[\begin{array}{c}
h_{1}(\lambda) \\
0 \\
0 \\
0
\end{array}\right]= & U\left[\begin{array}{c}
0 \\
0 \\
-h_{1}(\lambda) \cos \lambda \\
h_{1}(\lambda) \sin \lambda
\end{array}\right] \\
= & \int_{0}^{\pi}-h_{1}(\lambda) \cos \lambda\left(-J_{1}\right) d E_{\lambda} f_{k} \\
& +\int_{0}^{\pi} h_{1}(\lambda) \sin \lambda\left[\csc \lambda\left(-J_{1} \cos \lambda+J_{2}\right)\right] d E_{\lambda} f_{k} \\
= & \int_{0}^{\pi} h_{1}(\lambda) J_{2} d E_{\lambda} f_{k}
\end{aligned}
$$

and

$$
J_{2} U\left(\begin{array}{c}
h_{1}(\lambda) \\
0 \\
0 \\
0
\end{array}\right)=J_{2} \int_{0}^{\pi} h_{1}(\lambda) d E_{\lambda} f_{k}=\int_{0}^{\pi} h_{1}(\lambda) J_{2} d E_{\lambda} f_{k}
$$

Computing in a similar fashion for the other three components (and $J_{1}$ ) we obtain the desired equality. Done.

LEMMA 4.14. Let $\left\{A_{n}\right\}, n=1,2, \ldots$, be a sequence of bounded selfadjoint operators on a separable Hilbert space. Suppose there is an operator. A such that $A_{n} \rightarrow A$ in Hilbert-Schmidt norm. Then $\cos A_{n}, \sin A_{n}$ converge to $\cos A, \sin A$ in Hilbert-Schmidt norm respectively.

Proof. First observe

$$
\frac{d}{d t}\left(e^{\left.i t A^{-i t A_{n}}\right)}=i e^{i t A}\left(A-A_{n}\right) e^{-i t A_{n}}\right.
$$

integrating from 0 to 1 we obtain

$$
e^{i A} e^{-i A_{n}}-I=\int_{0}^{1} i e^{i t A}\left(A-A_{n}\right) e^{-i t A_{n}} d t
$$


Now

$$
\begin{aligned}
& \left\|e^{i A}-e^{i A_{n}}\right\|_{\mathrm{H} . S .}=\left\|\left(e^{i A}-e^{i A_{n}}\right) e^{-i A_{n}}\right\|_{\mathrm{H} . \mathrm{S} .}=\left\|e^{i A} e^{-i A_{n}}-I\right\|_{\mathrm{H} . S .} \\
& =\left\|\int_{0}^{1} i e^{i t A}\left(A-A_{n}\right) e^{-i t A_{n}} d t\right\|_{\mathrm{H} . \mathrm{S}} \leqslant \int_{0}^{1}\left\|e^{i t A}\left(A-A_{n}\right) e^{-i t A_{n}}\right\|_{\text {H.S. }} d t \\
& =\int_{0}^{1}\left\|A-A_{n}\right\|_{\text {H.S. }} d t=\left\|A-A_{n}\right\|_{\text {H.S. }}
\end{aligned}
$$

since Hilbert-Schmidt norm is preserved under multiplication by a unitary. Summarizing, for every $\varepsilon>0$, there is an $N$ such that

$$
\left\|e^{i A}-e^{-i A_{n}}\right\|_{\text {H.S. }}<\left\|A-A_{n}\right\|_{\text {H.S. }}<\varepsilon \text { for } n>N \text {. }
$$

It follows that $\left\|\cos A-\cos A_{n}\right\|_{\text {H.S. }}<\varepsilon,\left\|\sin A-\sin A_{n}\right\|_{\text {H.S. }}<\varepsilon$ for $n>N$. Done.

LEMMA 4.15. Let $J_{2}$ be an arbitrary complex structure on a real Hilbert space $(K, s)$ and $J_{1}$ be the complex structure associated with a paired basis of $(K, s)$ as in $\$ 1$. There is a sequence of complex structures $\left\{J_{2, n}\right\}, n=1,2, \ldots$, such that $-\left(J_{1}-J_{2, n}\right)^{2}$ has pure point spectrum and $\left\|J_{2}-J_{2, n}\right\|_{\text {H.s. }} \rightarrow 0$ as $n \rightarrow \infty$.

Proof. Consider $J_{2}^{\prime} \mid \mathcal{H}_{k}$ as above; as in the Weyl-von Neumann theorem we may approximate multiplication by $\lambda$ on $\mathcal{L}_{R}^{2}\left([0, \pi], \mu_{k}\right)$ by an operator $\Lambda_{k}$ with pure point spectrum such that $\left\|\Lambda_{k}-\lambda \cdot\right\|_{\text {H.s. }}<1 / 8 n 2^{k}$. By the proof of Lemma 4.14, $\left\|\cos \Lambda_{k}-\cos \lambda \cdot\right\|_{\text {H.S. }}<1 / 8 n 2^{k}$. Now define

with

$$
J_{2, n}^{\prime} \mid \mathcal{H}_{k}=\left(\begin{array}{cc}
0 & R \\
-R^{+} & 0
\end{array}\right)
$$

$$
R=\left(\begin{array}{cc}
\cos \Lambda_{k} & -\sin \Lambda_{k} \\
\sin \Lambda_{k} & \cos \Lambda_{k}
\end{array}\right) .
$$

Now $\left\|\left(J_{2, n}^{\prime}-J_{2}^{\prime}\right) \mid \mathcal{H}_{k}\right\|_{\text {H.S. }}<1 / n 2^{k}$, by straightforward computation. This yields

$$
\left\|J_{2, n}^{\prime}-J_{2}^{\prime}\right\|_{\text {H.S. }} \leqslant \sum_{k=0}^{\infty} \frac{1}{n 2^{k+1}}=\frac{1}{n} .
$$

Finally, define

$$
J_{2, n}\left|K_{2}=U J_{2}^{\prime} U^{*}, \quad J_{2, n}\right| K_{0}=J_{2}, \quad J_{2, n} \mid K_{1}=J_{2} .
$$

Then

$$
\left\|J_{2}-J_{2, n}\right\|_{\text {H.S. }}=\left\|\left(J_{2}-J_{2, n}\right) \mid K_{2}\right\|_{\text {H.S. }}=\left\|J_{2, n}^{\prime}-J_{2}^{\prime}\right\|_{\text {H.S. }}<1 / n \text {. }
$$

Further, $-\left(J_{1}^{\prime}-J_{2, n}^{\prime}\right)^{2}$ has pure point spectrum since $-\left(J_{1}^{\prime}-J_{2, n}^{\prime}\right)^{2} \mid \mathcal{H}_{K}=2 I-$ $2 \cos \Lambda_{k}$ and $\Lambda_{k}$ has pure point spectrum. From the unitarity of $U$ and the definition of $J_{2, n}$ on $K_{0}$ and $K_{1}$ we conclude $-\left(J_{1}-J_{2, n}\right)^{2}$ has pure point spectrum. Done. 
LEMMA 4.16. Let $\omega_{S, T}$ and $\omega_{S^{\prime}, T^{\prime}}$ be pure generalized free states of $\mathfrak{A}(\mathcal{K})$ such that $S-S^{\prime}$ is a Hilbert-Schmidt class operator. Then $\operatorname{Tr} T(I-T)=\infty \Leftrightarrow$ $\operatorname{Tr} T^{\prime}\left(I-T^{\prime}\right)=\infty$.

Proof. Equivalently, we show $\operatorname{Tr} T(I-T)<\infty \Leftrightarrow \operatorname{Tr} T^{\prime}\left(I-T^{\prime}\right)<\infty$. Observing that it is sufficient to prove $(\Rightarrow)$ we assume $\operatorname{Tr} T(I-T)<\infty$. From the proof of Proposition 4.5 we have

$$
\|S\|_{\mathrm{H} . \mathrm{S} .}^{2}=\|T\|_{\mathrm{Tr}}-\|T\|_{\mathrm{H} . \mathrm{S} .}^{2}=\operatorname{Tr} T(I-T) ;
$$

by hypothesis $\operatorname{Tr} T(I-T)<\infty$ and therefore

$$
\begin{aligned}
\left\|S^{\prime}\right\|_{\text {H.S. }} & \leqslant\|S\|_{\text {H.S. }}+\left\|S-S^{\prime}\right\|_{\text {H.S. }}<\infty \\
& \Rightarrow \operatorname{Tr} T^{\prime}\left(I-T^{\prime}\right)<\infty,
\end{aligned}
$$

since the above equality holds for $S^{\prime}, T^{\prime}$ as well. Done.

We are now ready to remove the point spectrum condition.

THEOREM 4.17. Let $\omega_{S, T}$ be a pure nongauge invariant generalized free state of $\mathfrak{U}(\mathcal{K})$. Then $\omega_{S, T}^{\circ}$ is a factor state $\Leftrightarrow \operatorname{Tr} T(I-T)=\infty$.

Proof. $(\Rightarrow)$ Suppose $\operatorname{Tr} T(I-T)<\infty$; then $T$ has pure point spectrum. In [7, Lemma 4.8] it is shown that if $T$ is a projection, $S=0$; from the definitions it follows that $\omega_{S, T}$ is gauge invariant. Hence, $T$ is not a projection and the proof reduces to that of Theorem 3.10.

$(\Leftarrow)$ Let $\omega_{J_{2}}, \omega_{J_{1}}$ be the states of $\mathfrak{A}(K, s)$ corresponding to $\omega_{S, T}, \omega_{0,0}$ respectively. As we noted in the proof of Corollary 3.6 if $M=-\left(J_{1}-J_{2}\right)^{2}$ has pure point spectrum, $T$ has pure point spectrum and we are done by Theorem 3.10. If not, by Lemma 4.15 there is a sequence of complex structures $J_{2, n}$ such that $-\left(J_{1}-J_{2, n}\right)^{2}$ has pure point spectrum and $\| J_{2}$ $J_{2, n} \|_{\text {H.S. }} \rightarrow 0$ as $n \rightarrow \infty$. By Proposition 4.5, we have $\left\|\omega_{J_{2, n}}-\omega_{J_{2}}\right\| \rightarrow 0$ as $n \rightarrow \infty$. Let $\omega_{S_{n}, T_{n}}$ be the state of $\mathfrak{U}(\mathcal{K})$ corresponding to $\omega_{J_{2, n}}$, then

$$
\left\|\omega_{S_{n}, T_{n}}^{\circ}-\omega_{S, T}^{\circ}\right\| \leqslant\left\|\omega_{S_{n}, T_{n}}-\omega_{S, T}\right\|=\left\|\omega_{J_{2, n}}-\omega_{J_{2}}\right\| \rightarrow 0
$$

as $n \rightarrow \infty$. By Lemmas 4.16, 4.4 and Theorem 3.10, the $\omega_{S_{n}, T_{n}}^{\circ}$ are also factor states. Thus $\omega_{S, T}^{\circ}$ is the uniform limit of factor states, hence a factor state. Done.

We conclude with a

REMARK. If $\omega_{S, T}$ is a pure gauge invariant state of $\mathfrak{U}(\mathcal{K})$ it follows from [10, III.1, Proposition] that $\omega_{S, T}^{\circ}$ is pure.

\section{REFERENCES}

1. H. Araki, On quasifree states of CAR and Bogoliubov automorphisms, Publ. Res. Inst. Math. Sci. Ser. A 6 (1970), 385-442.

2. B. M. Baker, Free states of the gauge invariant canonical anticommutation relations, Trans. Amer. Math. Soc. 237 (1978), 35-61. 
3. E. Balslev, J. Manuceau and A. Verbeure, Representations of anticommutation relations and Bogoliuboo transformations, Comm. Math. Phys. 8 (1968), 315-326.

4. O. Bratteli, Inductive limits of finite dimensional C*-algebras, Trans. Amer. Math. Soc. 171 (1972), 195-234.

5. J. Glimm, On a certain class of operator algebras, Trans. Amer. Math. Soc. 95 (1960), 318-340.

6. R. Haag, The mathematical structure of the Bardeen-Cooper-Schrieffer model, Nuovo Cimento 25 (1962), 287-299.

7. R. T. Powers, Thesis, Princeton University, Princeton, N. J., 1967.

8. __ Representations of uniformly hyperfinite algebras and their associated von Neumann rings, Ann. of Math. 86 (1967), 138-171.

9. R. T. Powers and E. Stormer, Free states of the canonical anticommutation relations, Comm. Math. Phys. 16 (1970), 1-33.

10. G. Stamatopoulos, Thesis, University of Pennsylvania, Philadelphia, Pa., 1974.

11. A. Van Daele and A. Verbeure, Unitary equivalence of Fock representations on the Weyl algebra, Comm. Math. Phys. 20 (1971), 268-278.

Department of Mathematics, Dalhousit Universtty, Halifax, Nova SCOtia, Canada

Current address: Department of Mathematics, State University of New York, Center at Buffalo, Buffalo, New York 14214 